\title{
LINE ASYMMETRY AND EXCITATION MECHANISM OF SOLAR OSCILLATIONS
}

\author{
R. NIGAM, A.G. KOSOVICHEV, P.H. SCHERRER AND J. SCHOU \\ W.W. Hansen Experimental Physics Laboratory, \\ Stanford University, Stanford CA 94305, U.S.A.
}

\section{Introduction}

In his opening address at the conference Dr. Tim Brown posed the line asymmetry problem between velocity and intensity as a puzzle in helioseismology that has been resisting theoretical explanation for many years. It was the observations of Duvall et al. (1993) that for the first time indicated that the power spectrum of solar acoustic modes show varying amounts of asymmetry. In particular, the velocity and intensity power spectra revealed an opposite sense of asymmetry. Many doubted the correctness of the experiment and thought it to be a puzzling result (Abrams \& Kumar, 1996). Many authors have investigated this problem theoretically and have found that there is an inherent asymmetry whenever there is a localized source exciting the solar oscillations (Gabriel, 1995; Roxburgh \& Vorontsov, 1995; Abrams \& Kumar, 1996; Nigam et al. 1997). This problem has important implications in helioseismology where the eigenfrequencies are generally determined by assuming that the power spectrum was symmetric and can be fitted by a Lorentzian. This leads to systematic errors in the determination of frequencies and, thus, affects the results of inversions (Rhodes et al. 1997). In this paper we offer an explanation for the difference in parity of the two asymmetries and estimate the depth and type of the sources that are responsible for exciting the solar p-modes.

\section{Observations}

Power spectra are computed from the full disk velocity and intensity images obtained from the MDI instrument (Scherrer et al. 1995). From these two power spectra shown in Figure 1A we see that the p-mode peaks of the velocity spectrum have negative asymmetry (more power on the low frequency end of the peak) while the peaks of the intensity spectrum have positive asymmetry (more power on the high frequency end of the peak). In the velocity spectrum (Figure $1 \mathrm{~A}(\mathrm{a})$ ), the asymmetry is strongest for low frequency (low radial order) modes and becomes negligible around and above the acoustic cut-off frequency $(\approx 5.2 \mathrm{mHz})$. However, the asymmetry in the intensity oscillations (Figure 1A(b)) increases with frequency for modes below the acoustic cut- 

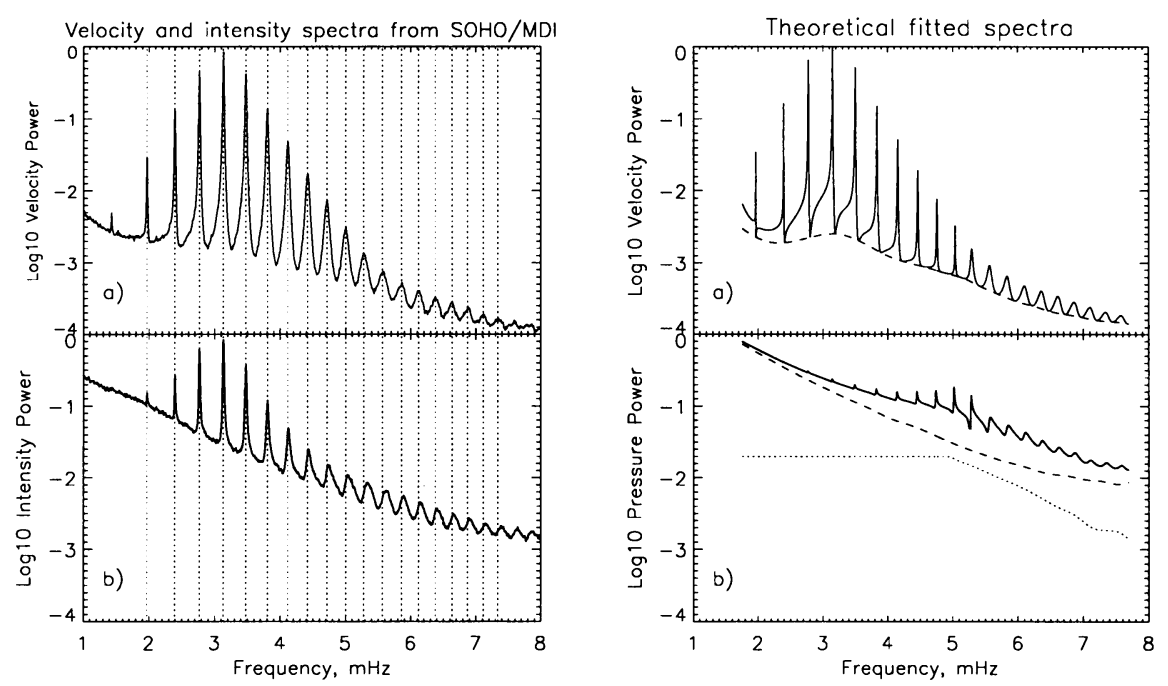

Figure 1. A. Normalized power spectra for solar oscillations of angular degree $l=200$ : a) Doppler velocity and b) continuum intensity for the same 3-day period of 21-23 July 1997 . The leftmost peak of the velocity spectrum corresponds to the f-mode. The other peaks of both spectra correspond to acoustic (p) modes of radial order from 1 to 21 (from the left to the right). The vertical dotted lines in both panels indicate the locations of the p-mode maxima in the velocity power spectrum to show that a relative shift in frequency occured at and above the acoustic cut-off frequency $(\approx 5.2 \mathrm{mHz})$.

B. Normalized theoretical power spectra for solar p-modes of $l=200$ produced by a composite source located at a depth, $d=75 \mathrm{~km}$ beneath the photospheric level and an observing location $r_{\text {obs }}=300 \mathrm{~km}$ above the photosphere, where the observed spectral line is formed: a) velocity spectrum (solid line) with additive uncorrelated noise (dashed line) and b) pressure spectrum (solid line) with additive correlated (dotted line) and uncorrelated noise (dashed line).

off frequency, and then gradually decreases at higher frequencies. We find in the data that the f-mode shows a similar reversal of asymmetry as in the case of p-modes.

\section{Numerical model of solar p-mode excitation}

Physically, solar acoustic modes are formed by resonances of the acoustic cavity beneath the Sun's surface. The shape and size of the cavity depends on the soundspeed and density stratification and on the mode angular degree. We assume that these waves are generated by turbulence in the convection zone, and apply Lighthill's (1952) method, in which the acoustic sources of various multipole orders are transferred to the right hand side of the wave equation, to calculate the velocity and pressure perturbations. We also assume that the intensity variations recorded by the MDI instrument correspond to Lagrangian pressure (or temperature) perturbations. Then, using a standard decomposition onto spherical harmonics (Gough, 1993) we 
transform Lighthill's equations into a single second-order wave equation

$$
\frac{d^{2} \Psi}{d r^{2}}+\left[\frac{\omega^{2}-\omega_{c}^{2}}{c^{2}}-\frac{L^{2}}{r^{2}}\left(1-\frac{N^{2}}{\omega^{2}}\right)\right] \Psi=S[f, q]
$$

where $\Psi$ is proportional to the Lagrangian pressure perturbation $\delta p, r$ is the radius, $\omega$ is the frequency, $\omega_{c}$ is the acoustic cut-off frequency, $c$ is the equilibrium sound speed, $N$ is the equilibrium buoyancy frequency, $L=\sqrt{l(l+1)}, S$ is a combination of source terms that include the fluctuating Reynolds stress force (dipole source term), $\boldsymbol{f}$ and the mass source (monopole term), $q$. The Green's function $G_{\Psi}\left(r, r_{\mathrm{s}}\right)$ of equation (1) using a standard solar model of Christensen-Dalsgaard el al. (1996), is found numerically with the boundary conditions that $G_{\Psi}=0$ at $r=0$ (as the perturbations are negligible much below the lower turning point) and the Sommerfeld radiation condition is applied above the photosphere (this ensures outgoing waves). Damping is added by making the frequency complex.

\section{Why is the asymmetry reversed?}

The Green's function for the pressure perturbation is calculated from equation (1). The Lagrangian perturbations are then calculated from the Green's function for a single source location and source type by multiplying by a suitable source function and adding solar noise. The noise is assumed to consist of a part $c_{\mathrm{p}}(\nu)$ that is correlated with the source function $s(\nu)$, while $n_{\mathrm{p}}(\nu)$ forms the uncorrelated additive background. One then obtains for the pressure perturbation

$$
p(\nu)=s(\nu)\left[c_{\mathrm{p}}(\nu)+G_{\mathrm{p}}(\nu)\right]+n_{\mathrm{p}}(\nu)
$$

and the velocity perturbation is found in a similar manner, but the correlated part here is much smaller. Equation (2) is a simple model of the observed solar signal, that includes the correlated noise. Here $G_{\mathrm{p}}$ is proportional to $G_{\Psi}$. The asymmetry in velocity and intensity power spectra is of opposite sense because a component of the solar noise that is correlated with the source is present. The correlated component of the noise is below a certain threshold to preserve the asymmetry obtained by the above model in the velocity power spectrum but that it is large enough (the correlated component is above a certain threshold) to reverse the asymmetry in the intensity spectrum. The source position $r_{\mathrm{s}}$ from the origin is kept fixed and the power spectrum is computed from equation (2) for the pressure perturbation. It is found that the correlation $c_{\mathrm{p}}(\nu)$ reverses the asymmetry found in $G_{\mathrm{p}}(\nu)$ when computing the power spectrum as seen from Figure $1 \mathrm{~B}(\mathrm{~b})$. The uncorrelated noise plays no role in the reversal of asymmetry. The intensity and velocity fluctuations are computed from the absorption line that is formed at a particular height in the solar atmosphere. The spectral line is effected by the p-mode oscillations and the solar granulation, which modulate and shift the line. It is thus the granulation overshoot that forms the correlated component of the noise as it transports the effect of the source directly onto the line in the atmosphere. It also excites the solar oscillations in the intergranular lanes (Goode et al., these Proceedings). Without adding correlated noise no reversal in asymmetry can be brought about between intensity and velocity. 


\section{Results and discussions}

The velocity and pressure power spectra computed from equations (1) and (2) are shown in Figure 1B. They capture the features of the asymmetry in the observations (Figure 1A). It is important to note that the narrow range of the acoustic source depth $(75 \pm 50 \mathrm{~km})$ found by comparing the theoretical and observed spectra coincides with the region of superadiabatic convection in the solar model. This region represents the highly unstable upper boundary layer of the convection zone where the convective motions are most violent. At this conference recent observations of Goode together with the simulations of Nordlund suggest that the p-modes are excited in the cooler intergranular lanes. Their data also indicates that the solar noise is correlated to the acoustic source. This might be a plausible explanation for the reversal of asymmetry between velocity and intensity power spectra.

\section{Conclusion}

In the talk a solution to the puzzle was presented. From our model we see that the intensity and velocity power spectra have opposite sense of asymmetry, because a correlated component of the solar noise is present in the data. A composite (monopole and dipole) source that excites the solar p-modes is found to be located in a thin superadiabatic layer $75 \pm 50 \mathrm{~km}$ below the photosphere.

We thank Tom Duvall, Douglas Gough, Åke Nordlund, Stuart Jefferies, Sergei Vorontsov and Ron Bracewell for useful discussions. SOHO is a project of international cooperation between ESA and NASA. This research is supported by the SOI-MDI NASA contract NAG5-3077 at Stanford University.

\section{References}

Abrams, D. and Kumar, P. 1996, Astrophys. J. 272, 882

Christensen-Dalsgaard, J. and GONG Team 1996, Science. 272, 1286

Duvall, T.L. Jr., Jefferies, S.M., Harvey, J.W., Osaki, Y. and Pomerantz, M.A. 1993, Astrophys. J. 410, 829

Gabriel, M. 1995, Astr. Astrophys. 299, 245

Gough, D.O. 1993, in: Astrophysical Fluid Dynamics, (ed. J.P. Zahn and J. Zinn-Justin, Elsevier, Amsterdam), 399-560

Lighthill, M.J. 1952, Proc. Roy. Soc. Lon. A211, 564

Nigam, R., Kosovichev, A.G. and Scherrer, P.H. 1997, in Sounding Solar and Stellar Interiors, Proc. IAU Symp. 181, in press

Nigam, R., Kosovichev, A.G., Scherrer, P.H. and Schou, J. 1997, ApJL., submitted

Rhodes, E.J. Jr., Kosovichev, A.G., Schou, J., Scherrer, P.H. and Reiter, J. 1997, Solar Phys. 175 , in press

Roxburgh, I.W. and Vorontsov, S.V. 1995, MNRAS. 272, 850

Scherrer, P.H. and MDI Team 1995, Solar Phys. 162, 129 